\title{
Tuning crystallographic compatibility to enhance shape memory in ceramics
}

\author{
Justin Jetter $\odot,{ }^{1, *}$ Hanlin Gu, ${ }^{2, *}$ Haolu Zhang, ${ }^{3, *}$ Manfred Wuttig, ${ }^{4}$ Xian Chen, ${ }^{5}$ Julia R. Greer, ${ }^{3}$ \\ Richard D. James $\odot,{ }^{2}$ and Eckhard Quandt ${ }^{1, \dagger}$ \\ ${ }^{1}$ Institute for Materials Science, Faculty of Engineering, University Kiel, Kaiserstraße 2, 24143 Kiel, Germany \\ ${ }^{2}$ Department of Aerospace Engineering and Mechanics, University of Minnesota, Minneapolis, Minnesota 55455, USA \\ ${ }^{3}$ Division of Engineering and Applied Science, California Institute of Technology, Pasadena, California 91125, USA \\ ${ }^{4}$ Department of Materials Science and Engineering, University of Maryland, College Park, Maryland 20742, USA \\ ${ }^{5}$ Mechanical and Aerospace Engineering, The Hong Kong University of Science and Technology, Clear Water Bay, Hong Kong
}

(Received 17 July 2019; published 23 September 2019)

\begin{abstract}
The extraordinary ability of shape-memory alloys to recover after large imposed deformation motivates efforts to transpose these properties onto ceramics, which would enable practical shape-memory properties at high temperatures and in harsh environments. The theory of mechanical compatibility was utilized to predict promising ceramic candidates in the system $\left(\mathrm{Y}_{0.5} \mathrm{Ta}_{0.5} \mathrm{O}_{2}\right)_{1-x}-\left(\mathrm{Zr}_{0.5} \mathrm{Hf}_{0.5} \mathrm{O}_{2}\right)_{x}, 0.6<x<0.85$. When these compatibility conditions are met, a reduction in thermal hysteresis by a factor of 2.5 , a tripling of deformability, and a $75 \%$ enhancement in strain recovery within the shape-memory effect was found. These findings reveal that predicting and optimizing the chemical composition of ceramics to attain improved crystallographic compatibility is a powerful tool for enabling and enhancing their deformability that could ultimately lead to a highly reversible oxide ceramic shape-memory material.

DOI: 10.1103/PhysRevMaterials.3.093603
\end{abstract}

\section{INTRODUCTION}

Shape memory (SM) and the related effect of superelasticity occur in all classes of materials: metals [1], ceramics [2], and polymers [3]. Some crystalline solids display these effects with almost perfect shape recovery through a reversible firstorder phase transformation between a low-crystallographicsymmetry low-temperature phase (martensite) and a highersymmetry high-temperature phase (austenite). Extensive investigations of such phase transformations have deepened the fundamental understanding of shape-memory effect in metallic SM alloys and facilitated a variety of emerging applications; for example, NiTi-based SM alloys are now used as brain stents [4] and devices for minimally invasive surgery [5].

Properties of SM alloys depend significantly on the reversibility of the underlying phase transformation. It has been shown in metals that the transformation hysteresis decreases [6] and fatigue properties improve [7] dramatically with greater kinematic compatibility between the transforming phases. The compatibility conditions depend on the transformation stretch tensor [8], which can be directly calculated from the lattice parameters of austenite and martensite. Tuning lattice parameters by changing composition allows for optimizing macroscopic properties, i.e., hysteresis and functional fatigue. It has been shown that the middle eigenvalue, $\lambda_{2}$, of the positive-definite symmetric transformation stretch tensor equals unity; it brings about perfect compatibility between a single variant of martensite and the (unstressed)

\footnotetext{
*These authors contributed equally to this work.

†Corresponding author: eq@tf.uni-kiel.de
}

austenite phase [9]. As $\lambda_{2}$ approaches 1, the elastic energy in the usual stressed transition layer at the habit plane between austenite and twinned martensite vanishes [10] and this has a dramatic effect on thermal hysteresis. This condition of $\lambda_{2}=1$ is first in a hierarchy of compatibility conditions that allow additional unstressed microstructures between the two phases. The strongest known conditions of compatibility are the so-called cofactor conditions, which include $\lambda_{2}=1$, as well as two additional restrictions and an inequality [11]. These conditions imply that many unstressed interfaces between phases become possible, which are either absent or stressed in common SM materials; this feature is termed supercompatibility [11,12].

These developments have significantly improved our understanding of metallic shape-memory alloys (SMAs). For example, the technologically important Ti-rich $\mathrm{NiTiCu}(\mathrm{Co})$ SMAs feature excellent compatibility (middle eigenvalue $\lambda_{2}=1.0008$, cofactors $C C 1=4.26 \times 10^{-5}, C C 2=3.43 \times$ $10^{-5}$ ) that helped reveal this alloy's low superelastic stress hysteresis of $50 \mathrm{MPa}$ and its resistance to fatigue-induced degradation: 10 million tension-stress-induced transformation cycles did not affect the transformation characteristics [13]. Another alloy discovered through using the cofactor theory, $\mathrm{Zn}_{45} \mathrm{Cu}_{25} \mathrm{Au}_{30}$, exhibits extremely small thermal hysteresis of $0.2{ }^{\circ} \mathrm{C}$ and nearly full repeatability after 100000 full transformation cycles at $7 \%$ strain and $700 \mathrm{MPa}$ compression stress cycles [14].

\section{CERAMIC SHAPE MEMORY}

Two-phase ceramic shape-memory materials were actively investigated until the 1990s, which led to the discovery of now well-known transformation toughened ceramics [15]. 
TABLE I. Three possible transformation mechanisms for tetragonal-to-monoclinic transformation in the (Y, Ta) $\mathrm{O}_{2}-(\mathrm{Zr}, \mathrm{Hf}) \mathrm{O}_{2}$ system.

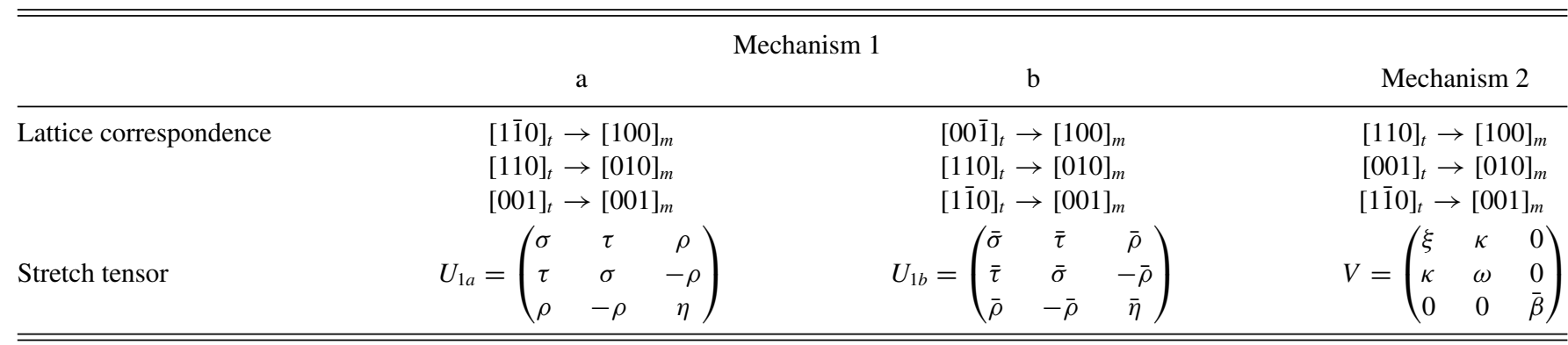

In the important class of $\mathrm{ZrO}_{2}$-based structural ceramics, metastable tetragonal homoprecipitates transform to the monoclinic phase in the stress field of a crack, blunt further crack propagation, and inhibit failure. This line of research stagnated as the materials approached their technological limits, with very limited efforts dedicated to developing shapememory ceramics that display metal-like shape-memory properties [16]. Potential shape-memory ceramics display relatively large thermal hysteresis [17] when compared to many metallic shape-memory alloys. Various approaches have been used to change the transformation temperatures of matrix and particles, e.g., solid solutions formed with $\mathrm{CeO}_{2}$ [18] or stabilization by $\mathrm{Y}_{2} \mathrm{O}_{3}$ [19]. The superelastic and shape-memory behavior was demonstrated in freestanding $\mathrm{CeO}_{2}-\mathrm{ZrO}_{2}$ pillars of approximately $1 \mu \mathrm{m}$ diameter [2]. This experiment proved that ceramics possess all the necessary requirements for a shape-memory effect, but the low compatibility rooted in these $\mathrm{ZrO}_{2}$-based ceramics is much larger than that found in most metallic SM alloys. This leads to an extremely large stress hysteresis that exceeds $1 \mathrm{GPa}$ so that any shapememory-like properties in ceramics are currently of limited use [2]. Here, we show that the success in improving the repeatability and lowering of the superelastic hysteresis and shape-memory effects in metallic SMAs by satisfying conditions of compatibility can be translated to oxide ceramics.

We apply compatibility theory to a systematic investigation of structural ceramics in the system $\left(\mathrm{Y}_{0.5} \mathrm{Ta}_{0.5} \mathrm{O}_{2}\right)_{1-x}-\left(\mathrm{Zr}_{0.5} \mathrm{Hf}_{0.5} \mathrm{O}_{2}\right)_{x}$, with $0.6<x<0.85$; a transformation of the tetragonal into a monoclinic phase had already been identified for $\left(\mathrm{Y}_{0.5} \mathrm{Ta}_{0.5} \mathrm{O}_{2}\right)_{1-x}-\left(\mathrm{ZrO}_{2}\right)_{x}$ at $0.6<x<1$ [16]. We focused on $\mathrm{ZrO}_{2}-\mathrm{HfO}_{2}$ solid solutions because the crystal structures, chemical behavior, and lattice parameters of $\mathrm{ZrO}_{2}$ and $\mathrm{HfO}_{2}$ are virtually identical. $\mathrm{HfO}_{2}$ has a higher transition temperature that stabilizes the monoclinic phase at higher temperatures and provides the first-order phase transition at higher doping levels [17]. We demonstrate a close correlation between $\left|\lambda_{2}=1\right|$ and hysteresis in this promising oxide system, and present a comparison of stress-induced variant rearrangement and the shape-memory effect in low- and high-hysteresis samples.

\section{TRANSFORMATION MECHANISM}

The optimization of the complex phase-transforming (Y, Ta $) \mathrm{O}_{2}-(\mathrm{Zr}, \mathrm{Hf}) \mathrm{O}_{2}$ system begins with the identification of the transformation mechanism so that the transformation stretch tensor, and hence the middle eigenvalue $\lambda_{2}$ as well as the twinning parameters, can be computed. This information was used as a tuning guide towards our search for the lowesthysteresis ceramic shape-memory material in this system. Given the measured lattice parameters and symmetries for parent and child phases, we exploit an algorithm [8] that finds the proper lattice correspondence and delivers the corresponding stretch tensor with the least transformation strain (measured by a suitable norm). We identified three mechanisms that are associated with low transformation strains, as shown in Table I. The existence of multiple lattice correspondences in a low-symmetry system is supported by the findings of Hayakawa et al. [20,21] for the $\mathrm{t} \rightarrow \mathrm{m}$ transformation in $\mathrm{ZrO}_{2}-2$ mol. $\% \mathrm{Y}_{2} \mathrm{O}_{3}$.

The lengthy expressions for $\sigma, \tau, \eta$, etc., in terms of standard lattice parameters are given in the Supplemental Material [22]. For each of these cases there are four variants of the monoclinic phase and all pairs of variants can be twinned. The calculated values of $\lambda_{2}$ from mechanisms $1 \mathrm{a}$ and $1 \mathrm{~b}$ are the same due to a special symmetry arising from this tetragonal-to-monoclinic phase transformation [22].

We use the conventional notation for austenite start $\left(A_{s}\right)$, austenite finish $\left(A_{f}\right)$, martensite start $\left(M_{s}\right)$, and martensite finish $\left(M_{f}\right)$ temperatures. The measurement of transformation start and finish temperatures was performed by differential thermal analysis (DTA) and differential scanning calorimetry (DSC) (see the Supplemental Material [22]). Figure 1(a) shows that $A_{s}$ and $M_{s}$ increase with $x$ in the system $\left(\mathrm{Y}_{0.5} \mathrm{Ta}_{0.5} \mathrm{O}_{2}\right)_{1-x}-\left(\mathrm{Zr}_{0.5} \mathrm{Hf}_{0.5} \mathrm{O}_{2}\right)_{x}$ and that a compositional range exists where they approach each other. At those concentrations, the hysteresis $\left(\Delta T=0.5\left|\left(A_{f}+A_{s}\right)-\left(M_{s}+M_{f}\right)\right|\right)$ becomes considerably smaller than at other compositions with a minimum of approximately $120 \mathrm{~K}$ for a concentration of $x \approx 0.73$. This value means a reduction of the hysteresis of a factor of 2.5 between the maximum and minimum hysteresis in the compositional range $0.6<x<0.85$.

In view of the multiple likely transformation mechanisms, the analysis of the drop of hysteresis requires care. We show in Fig. 1(b) the values of both mechanisms 1 (open squares) and 2 (solid squares). By comparison of Figs. 1(a) and 1(b), we see that the lowest value of hysteresis at $x \approx 0.73$ is close to the crossing point where the maximum deviation of $\lambda_{2}$ from 1 is smallest (see Supplemental Material item 3 for detail on how to extract lattice parameters for $\lambda_{2}$ calculation [22]). This finding is consistent with the quantitative theory for the influence of $\lambda_{2}$ on hysteresis [10]. That theory is based on the analysis of an energy barrier to initiate the growth of a twinned platelet in the low-temperature phase. When the 

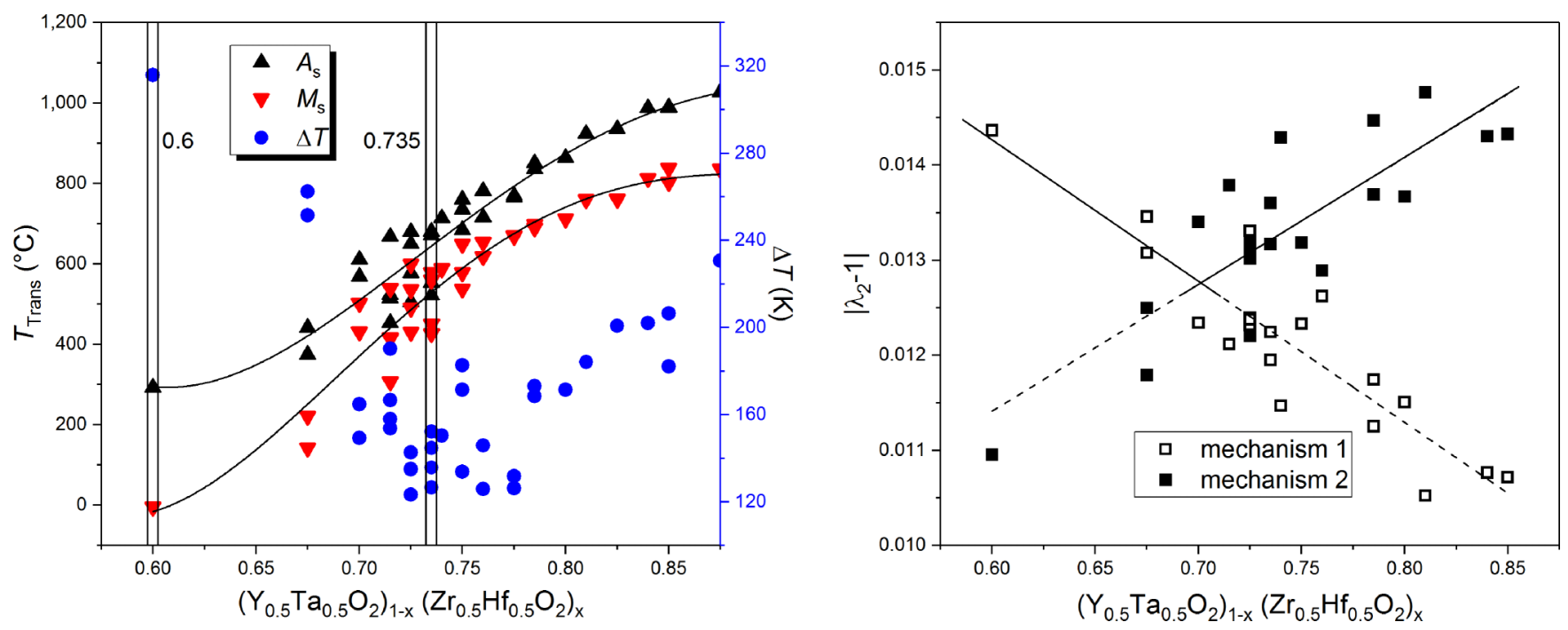

FIG. 1. (a) Transformation temperatures in the system $\left(\mathrm{Y}_{0.5} \mathrm{Ta}_{0.5} \mathrm{O}_{2}\right)_{1-x}-\left(\mathrm{Zr}_{0.5} \mathrm{Hf}_{0.5} \mathrm{O}_{2}\right)_{x}$ for $0.6<x<0.85$ between monoclinic and tetragonal phase $\left(A_{s}\right)$, as well as the temperatures for the reverse transformation $\left(M_{s}\right)$ with trend lines for different doping concentrations. The thermal hysteresis $\Delta T=0.5\left|\left(A_{f}+A_{s}\right)-\left(M_{s}+M_{f}\right)\right|$ is shown in blue with a minimum value of approximately $120 \mathrm{~K}$ at $x \approx 0.73$. Mechanical experiments were conducted on high-hysteresis samples $(x=0.6)$ and low-hysteresis samples $(x=0.735)$. (b) $\left|\lambda_{2}-1\right|$, which describes the distance from the optimal value of $\lambda_{2}=1$ for the two mechanisms which satisfy this condition the closest in the system $\left(\mathrm{Y}_{0.5} \mathrm{Ta}_{0.5} \mathrm{O}_{2}\right)_{1-x}-\left(\mathrm{Zr}_{0.5} \mathrm{Hf}_{0.5} \mathrm{O}_{2}\right)_{x}$ for $0.6<x<0.85$. The lines indicate the trend of the case with higher values. The crossing of those lines is close to the concentration that shows the lowest thermal hysteresis for its transformation.

platelet is small, interfacial energy dominates, leading to an increase of energy vs size. As the platelet grows, the twin density changes and a barrier is overcome when the bulk energy begins to dominate. The critical size of the inclusion at the barrier is extremely sensitive to the value of $\lambda_{2}$. Our results strongly suggest that it is the larger of the barriers of the two mechanisms that dominates the behavior (mechanism 1 for high-hysteresis and mechanism 2 for low-hysteresis samples), at least in the present low-symmetry system.

Another important observation relates to the numbers of twin systems. We modeled all twin systems of the phasetransforming samples for mechanisms 1 and 2 and discovered that for the lowest-hysteresis sample at $x \approx 0.73$, there exist 8 compatible austenite/martensite interfaces formed by mechanism 1 and 16 compatible austenite/martensite interfaces enabled by mechanism 2. Compared with the number of compatible austenite/martensite interfaces, samples with concentrations away from 0.73 consist of only one-half to threequarters of the compatible interfaces from both mechanisms.

The irregular contrast or fringes in the TEM bright-field images [Figs. 2(b) and 2(e)] are due to local changes in the diffraction quality, which can result from many sources such as thickness change, physical distortion, or material defects [23]. The fact that there is no clear periodicity in any one direction in the observed fringes suggests that it is most likely a result of heterogeneous lattice distortion, either bend contours or in-plane residual stresses. We indeed suspect that these distortions are consequences of imperfect compatibility during phase transformation, which inevitably cause residual strain in the martensite phase, since these strain fringes are similar to the contrast from lattice strain around defects in well-studied crystalline materials [24]. However, although the two images are taken using the same set of TEM settings, since the contrast is highly dependent on experimental conditions such as the diffraction error and beam convergence angle [25], no quantitative conclusion could be made solely based on the different contrast level in the two cases.

\section{MICROSTRUCTURE}

Further investigations of the twin systems were conducted under a transmission electron microscope (TEM). Figure 2(a) contains a TEM image of a martensite grain in a lowhysteresis sample $(x=0.735, \Delta T=120 \mathrm{~K})$ and reveals a twinning microstructure with laminates ranging from 40 to $500 \mathrm{~nm}$ in thickness. Figure 2(b) shows its bright-field (BF) image with a [001] zone axis in the martensite basis (as below), where the corresponding indexed diffraction pattern (inset) for the grain contains no twinning patterns, which suggests that all variants share the common two-fold axis [001]. No visible defects were observed within any variant, which indicates that the crystal is in a low-energy state with minimal internal stresses. The majority of twinning planes are found to be along (100)-, (010)-, and (110)-type planes. As the theory suggests, both mechanism 1 and mechanism 2 produce compatible austenite/martensite interfaces along (100) and (001), so the population of (100) twin planes supports the theoretical prediction that for $x=0.735$ the sample contains the maximum number of austenite/martensite interfaces. TEM images reveal that the mirror planes are mostly coherent, with an occasional incoherency, for example, the partial dislocation cutting through a (010) plane highlighted by an arrow in the high-resolution image in Fig. 2(c). TEM images of a high-hysteresis sample $(x=0.6, \Delta T=315 \mathrm{~K})$ reveal similar twinning microstructures at the near-micron scale shown in Fig. 2(d). Further investigation with BF at a higher resolution [Fig. 2(e)] shows that, although the twinning direction and mirror planes are identical to those in the 

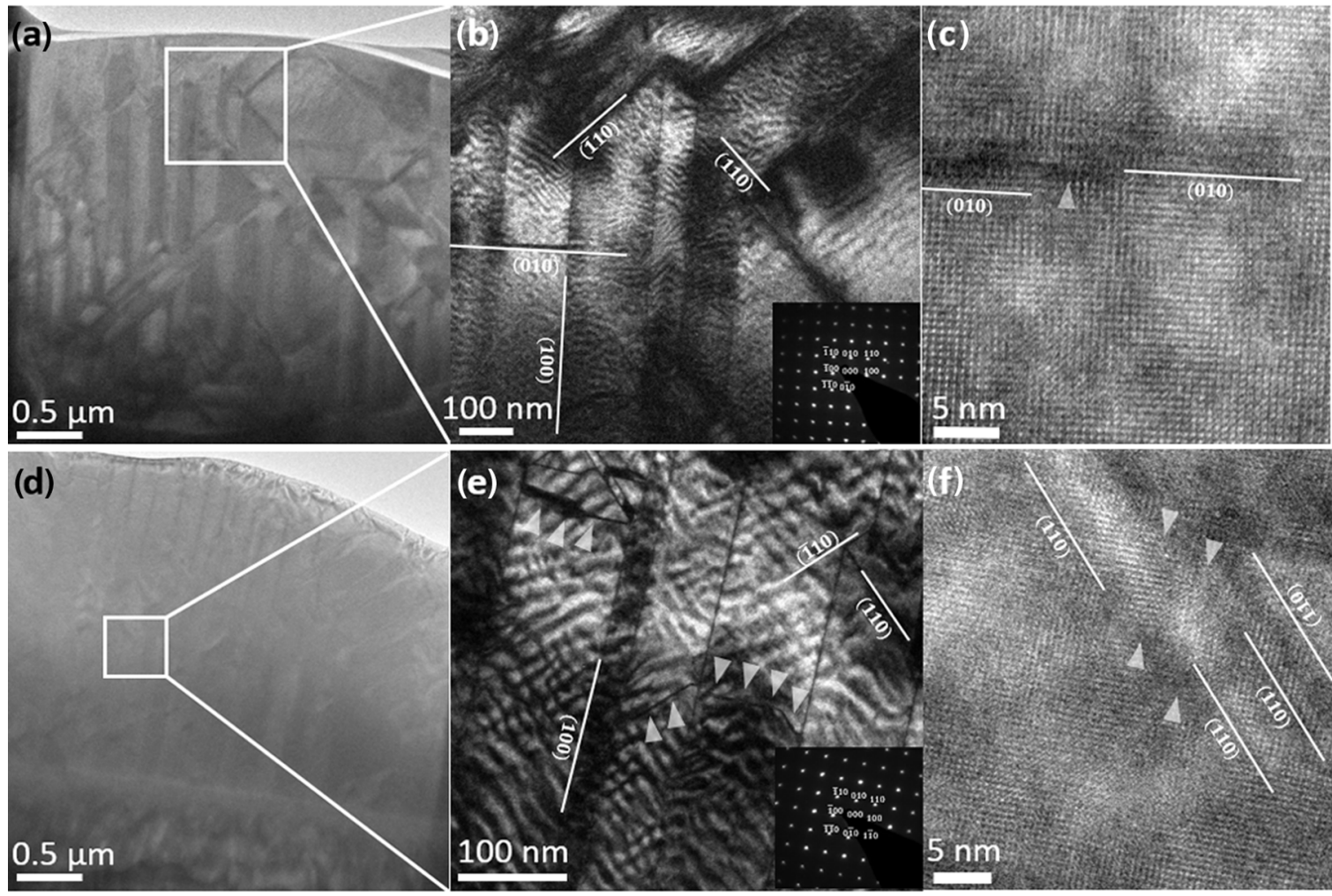

FIG. 2. (a) TEM image of a martensite grain from a low-hysteresis sample that reveals its complicated twinning microstructure with laminates as thin as $40 \mathrm{~nm}$. (b) A close-up BF image with an indexed diffraction pattern that reveals mirror planes of mainly (100), (010), and (110) type, and two-fold axes along the diffracting zone axis [001]. (c) High-resolution image of a coherent twin boundary intersected by a dislocation, indicated by the small arrow. (d-f) TEM images of a high-hysteresis sample that reveal similar microstructure and twin systems but populated with multiple defects within individual variants and at twin boundaries.

low-hysteresis system, the crystal is much more distorted and is populated with dislocations, which cut through individual variants (indicated by the small arrows; see also the Supplemental Material [22]). The high-resolution image of the high-hysteresis sample in Fig. 2(f) displays a complicated twin-boundary structure with multiple dislocations. This microstructural analysis supports that the high-hysteresis sample contains greater internal stress during phase transformation compared with the low-hysteresis system, as a result of its low compatibility.

Figure 3 shows stress-induced detwinning of the martensite phase, which is a prerequisite for shape memory and superelasticity [1]. Figure 3(a) demonstrates recoverable strain through martensite reorientation obtained from uniaxial compression of a typical single-crystalline 900-nm-diam pillar extracted using a focused ion beam (FIB) from the lowand high-hysteresis samples. The inset plot gives the crystallographic orientations along the loading direction using equal area projections from electron backscattered diffraction (EBSD; details in the Supplemental Material [22]). This plot reveals that both pillars favor the (100)[001] twin system: in the low-hysteresis sample, the onset of detwinning at about $800 \mathrm{MPa}$ is followed by a steady serrated flow at a constant applied stress, similar to the serrated detwinning stress plateau seen in the deformation of macroscopic metallic SMAs [26]. Unlike these previously studied systems, where the detwinning plateau is followed by a uniform nonlinear deformation due to incomplete reorientation [27,28], the detwinning process in this pillar ends with a rapid strain burst at the same stress, which suggests that the reorientation is completed at $5.9 \%$ strain. This strain burst and the subsequent stress drop at $6.3 \%$ are clear signatures of a dislocationmediated slip in small-scale plasticity $[29,30]$, followed by steep plastic hardening starting at 6.6\% strain. The highhysteresis sample undergoes a similar process after the first detwinning event at about $800 \mathrm{MPa}$ and has a significantly less recoverable strain from detwinning-it quickly transitions into the burst-governed plasticity at $1.3 \%$ strain, and begins to harden at $2.2 \%$ strain. This suggests that as the compatibility conditions become better satisfied, the postelastic-deformation mechanism transitions from a detwinning process to dislocation slip. This could be related to the preexistence of defects in the high-hysteresis system as shown in Fig. 2(f).

\section{SHAPE MEMORY}

The absence of steplike offsets on the pillar surface of Figs. 3(b) and 3(c) supports our conclusion that the strain during compression is largely carried out by detwinning and less from plastic slip $[31,32]$. The less pronounced shape change in the high-hysteresis pillar [Fig. 3(b)] compared to the low-hysteresis pillar [Fig. 3(c)] is indicative of lesser deformation from detwinning in the low-compatibility sample. The twin band structure, indicated by the yellow arrows, is oriented at approximately $30^{\circ}$ to the horizontal plane in the 

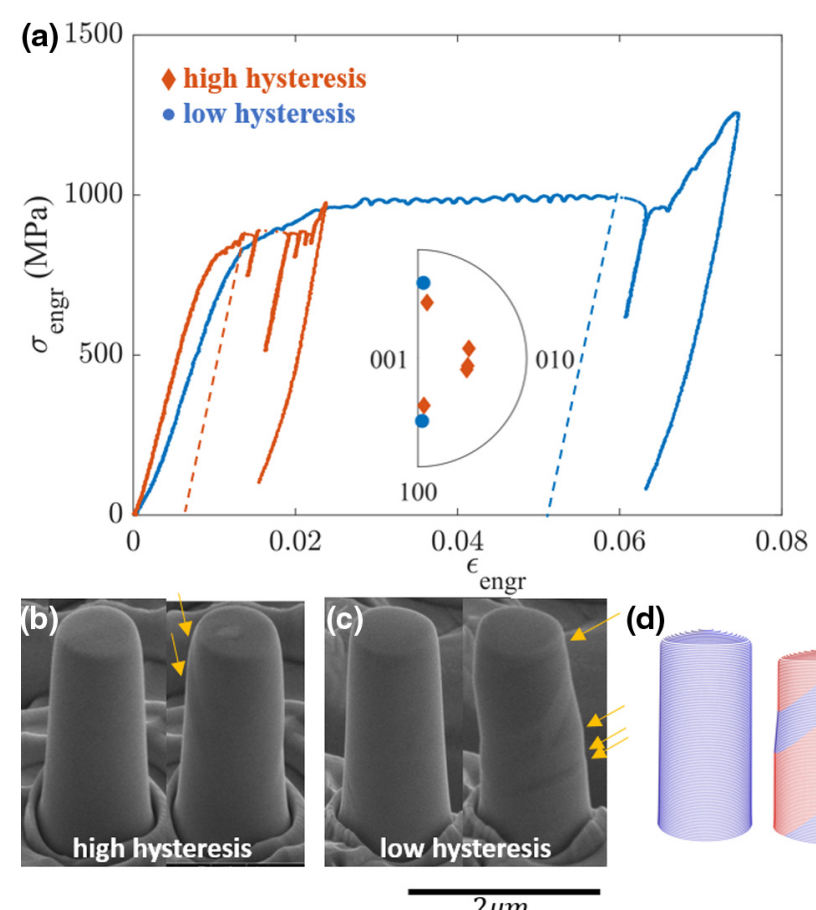

(d)

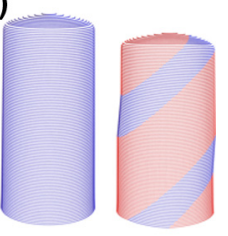

FIG. 3. (a) Stress-strain response of 900-nm-diam martensite pillars milled from low- and high-hysteresis samples, both with variant orientations that favor the (100)[001] twin system (inset plot shows the loading direction inverted pole figure for each pillar). Preand postcompression scanning electron microscopy (SEM) images of the pillars tested (a) carved out from a grain within the (b) highhysteresis and the (c) low-hysteresis phase. The boundaries between sheared variants are signified by the yellow arrows. (d) Theoretical prediction of the microstructure given by the Schmid law in the low-hysteresis case shows good agreement (5.9\% strain) with (c). low-hysteresis pillar, similar to that theoretically predicted in Fig. 3(d).

It is assumed that the pillar compression starts as a single variant of martensite that is compressed along [011] in a monoclinic lattice. Since the low-hysteresis sample achieves $6 \%$ reorientation strain, as shown in Fig. 3(a), we considered all possible twinning systems. The type-II twin between variants 1 and 4 in mechanisms $1 \mathrm{a}$ and $1 \mathrm{~b}$ is favored by the Schmid law and exhibits $5.9 \%$ strain by reorientation as in the experiment.

We also demonstrate the one-way shape-memory effect through experiments on irregularly shaped particles. Average deformation gradients $F$ were mapped using points on the particle whose spatial coordinates were found using image correlation, and the stretch tensors $U_{\text {comp }}$ and $U_{\text {heat }}$ were determined for each particle postcompression and postheating, using its original shape as the reference configuration (see the Supplemental Material [22] for details). We define the recovery of a particle as

$$
r=\frac{\left|U_{\text {comp }}-U_{\text {heat }}\right|}{\left|U_{\text {comp }}-I\right|} .
$$

A schematic of this experimental process is shown in Fig. 4(a), where each particle is first imaged in the SEM, compressed to a strain of $\left|U_{\text {comp }}-I\right|$, imaged again, heated to $100^{\circ} \mathrm{C}$ above its previously measured $A_{f}$, and then imaged again at room temperature. Figures 4(b)-4(d) provide SEM images of an irregularly shaped low-hysteresis particle at room temperature before compression by approximately $15 \%$ of the particle height, after compression, and after heating to $850^{\circ} \mathrm{C}$. To better visualize the shape recovery, the original shape of the particle is traced in white and overlaid on all three images, along with partial outlines of the particle after compression (red) and after heating (blue). These images reveal that
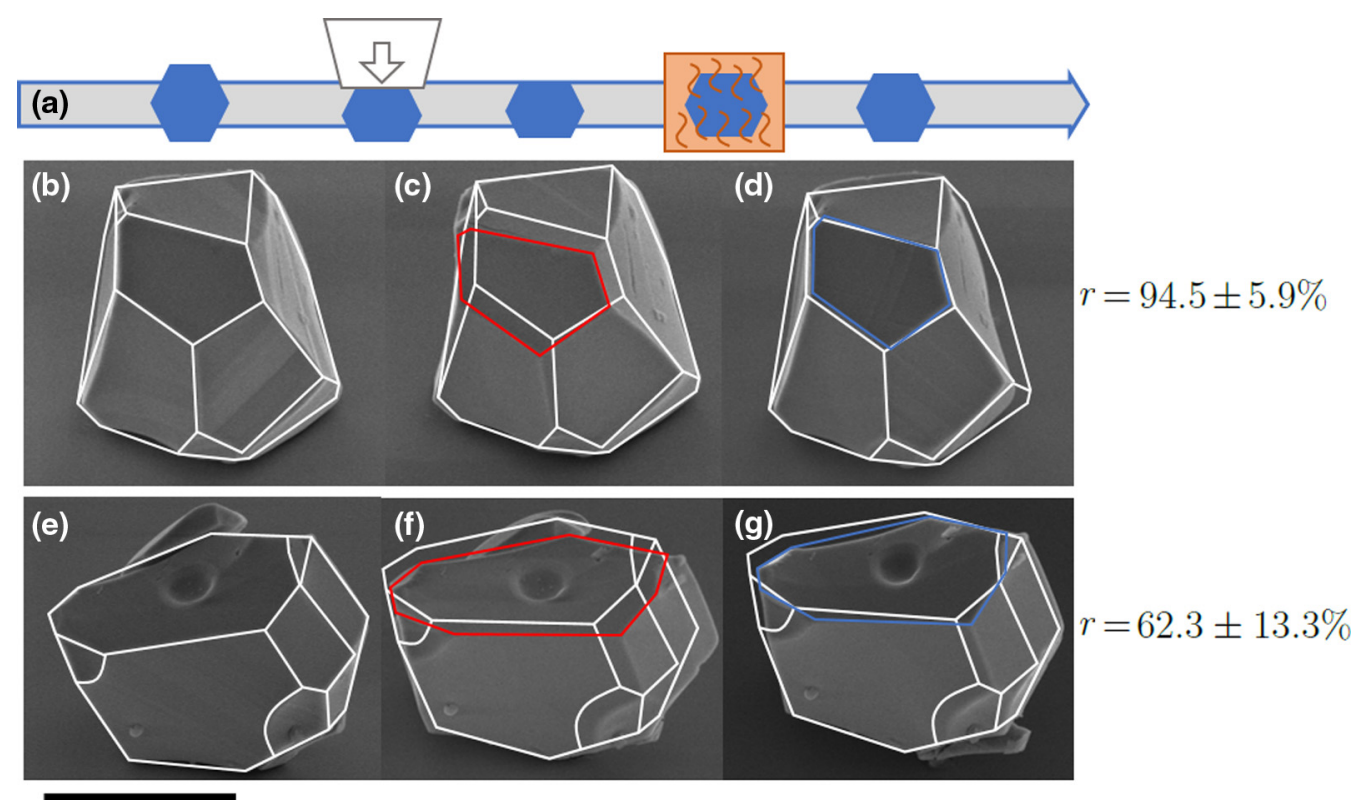

FIG. 4. (a) Schematic representation of the compression-heating cycle. The original shape of the particle is traced in white and overlaid on all three images, along with outlines of a selected face after compression (red) and after heating (blue). Images of a low-hysteresis particle (b) before compression, (c) after compression, and (d) after heating to $850^{\circ} \mathrm{C}$ and cooling to room temperature showing almost complete shape recovery. (e-g) Same process for a high-hysteresis particle showing that there is less recovery postheating. 
the low-hysteresis particle exhibits near-complete recovery [Figs. 4(b)-4(d)], while the high-hysteresis particle does not [Figs. 4(e)-4(g)]. We found the recovery $r$ to be $94.5 \pm$ $5.9 \%(N=5)$ for low-hysteresis particles and $62.3 \pm 13.3 \%$ $(N=3)$ for the high-hysteresis particles, proving that the low-hysteresis system exhibits near-complete shape recovery through the shape-memory effect.

\section{CONCLUSION}

In this paper we demonstrated that the theory of crystallographic compatibility provides an effective strategy to guide compositional changes for the discovery of reversible shape memory in ceramics. We performed nanomechanical experiments on site-specific, small-scale ceramic samples with predicted optimal composition to reveal the shape-memory effect. This comprehensive suite of theory, synthesis, in situ nanomechanical experiments, and microstructure analysis uncovered a new, unique class of potential shape-memory oxides. These new materials could provide major breakthroughs in vibration isolation and mitigation, deployable structures, and structural materials subjected to extreme thermomechanical environments.

\section{ACKNOWLEDGMENTS}

E.Q. and J.J. acknowledge support by the German Research Foundation (DFG) via a Reinhart Koselleck project Grant No. 313454214. J.R.G. gratefully acknowledges the financial support of the Stanback Space Innovation Program at Caltech and from the U.S. Department of Energy's Basic Energy Sciences through Grant No. DESC0016945. We also acknowledge H. Vo and P. Hosemann for providing their instrument for preliminary nanomechanical investigations. The work from the University of Minnesota was supported by NSF (Grant No. DMREF-1629026), ONR (Grant No. N00014-181-2766), and the MURI program (Grants No. FA9550-18-10095 and No. FA9550-16-1-0566). H.G. and R.D.J. are also pleased to acknowledge the support of Medtronic Corp, the Institute on the Environment (RDF fund), and the Norwegian Centennial Chair Program. X.C. thanks the financial support of the HK Research Grants Council under Grants No. 26200316 and No. 16207017. X.C. also thanks the Isaac Newton Institute for Mathematical Sciences for support and hospitality during the program "The Mathematical Design of New Materials" when work on this paper was undertaken by EPSRC Grant No. EP/R014604/1.
[1] K. Oksuka and C. M. Wayman, in Shape Memory Materials, edited by K. Otsuka and U.-C. C. M. Wayman (Cambridge University Press, Cambridge, UK, 1999) p. 284.

[2] A. Lai, Z. Du, C. L. Gan, and C. A. Schuh, Shape memory and superelastic ceramics at small scales, Science 341, 1505 (2013).

[3] M. Behl, M. Y. Razzaq, and A. Lendlein, Multifunctional shapememory polymers, Adv. Mater. 22, 3388 (2010).

[4] M. I. Chimowitz, M. J. Lynn, C. P. Derdeyn, T. N. Turan, D. Fiorella, B. F. Lane, L. S. Janis, H. L. Lutsep, S. L. Barnwell, M. F. Waters, B. L. Hoh, J. M. Hourihane, E. I. Levy, A. V. Alexandrov, M. R. Harrigan, D. Chiu, R. P. Klucznik, J. M. Clark, C. G. McDougall, M. D. Johnson, G. L. Pride, M. T. Torbey, O. O. Zaidat, Z. Rumboldt, and H. J. Cloft, Stenting versus aggressive medical therapy for intracranial arterial stenosis, N. Engl. J. Med. 365, 993 (2011).

[5] T. Duerig, A. Pelton, and D. Stöckel, An overview of nitinol medical applications, Mater. Sci. Eng. A 273-275, 149 (1999).

[6] J. Cui, Y. S. Chu, O. O. Famodu, Y. Furuya, J. Hattrick-Simpers, R. D. James, A. Ludwig, S. Thienhaus, M. Wuttig, Z. Zhang, and I. Takeuchi, Combinatorial search of thermoelastic shapememory alloys with extremely small hysteresis width, Nat. Mater. 5, 286 (2006).

[7] Y. Song, X. Chen, V. Dabade, T. W. Shield, and R. D. James, Enhanced reversibility and unusual microstructure of a phasetransforming material, Nature (London) 502, 85 (2013).

[8] X. Chen, Y. Song, N. Tamura, and R. D. James, Determination of the stretch tensor for structural transformations, J. Mech. Phys. Solids 93, 34 (2016).

[9] J. M. Ball and R. D. James, Fine phase mixtures as minimizers of energy, Arch. Ration. Mech. Anal. 100, 13 (1987).

[10] Z. Zhang, R. D. James, and S. Müller, Energy barriers and hysteresis in martensitic phase transformations, Acta Mater. 57, 4332 (2009).
[11] H. Gu, L. Bumke, C. Chluba, E. Quandt, and R. D. James, Phase engineering and supercompatibility of shape memory alloys, Mater. Today 21, 265 (2018).

[12] X. Chen, V. Srivastava, V. Dabade, and R. D. James, Study of the cofactor conditions: Conditions of supercompatibility between phases, J. Mech. Phys. Solids 61, 2566 (2013).

[13] C. Chluba, W. Ge, R. Lima de Miranda, J. Strobel, L. Kienle, E. Quandt, and M. Wuttig, Ultralow-fatigue shape memory alloy films, Science 348, 1004 (2015).

[14] X. Ni, J. R. Greer, K. Bhattacharya, R. D. James, and X. Chen, Exceptional resilience of small-scale $\mathrm{Au}_{30} \mathrm{Cu}_{25} \mathrm{Zn}_{45}$ under cyclic stress-induced phase transformation, Nano Lett. 16, 7621 (2016).

[15] M. V. Swain, Shape memory behaviour in partially stabilized zirconia ceramics, Nature (London) 322, 234 (1986).

[16] S. Shian, P. Sarin, M. Gurak, M. Baram, W. M. Kriven, and D. R. Clarke, The tetragonal-monoclinic, ferroelastic transformation in yttrium tantalate and effect of zirconia alloying, Acta Mater. 69, 196 (2014).

[17] C. Wang, M. Zinkevich, and F. Aldinger, The zirconia-hafnia system: DTA measurements and thermodynamic calculations, J. Am. Ceram. Soc. 89, 3751 (2006).

[18] M. Yashima, T. Mitsuhashi, H. Takashina, M. Kakihana, T. Ikegami, and M. Yoshimura, Tetragonal-monoclinic phase transition enthalpy and temperature of $\mathrm{ZrO}_{2}-\mathrm{CeO}_{2}$ solid solutions, J. Am. Ceram. Soc. 78, 2225 (1995).

[19] P. Li, I.-W. Chen, and J. E. Penner-Hahn, Effect of dopants on zirconia stabilization-an X-ray absorption study: I, trivalent dopants, J. Am. Ceram. Soc. 77, 118 (1994).

[20] M. Hayakawa, N. Kuntani, and M. Oka, Structural study on the tetragonal to monoclinic transformation in arcmelted $\mathrm{ZrO}_{2}-2 \mathrm{~mol} \% \mathrm{Y}_{2} \mathrm{O}_{3}$ - I. Experimental observations, Acta Metall. 37, 2223 (1989). 
[21] M. Hayakawa and M. Oka, Structural study on the tetragonal to monoclinic transformation in arc-melted $\mathrm{ZrO}_{2}-2 \mathrm{~mol} . \% \mathrm{Y}_{2} \mathrm{O}_{3}-$ II. Quantitative analysis, Acta Metall. 37, 2229 (1989).

[22] See Supplemental Material at http://link.aps.org/supplemental/ 10.1103/PhysRevMaterials.3.093603 for (1) a full expression of stretch tensor in terms of lattice parameters, (2) proof for mechanisms $1 \mathrm{a}$ and $1 \mathrm{~b}$ to be the same, (3) a description of how to measure and obtain accurate lattice parameters, (4) details on the DTA and DSC measurement procedure, (5) observation of dislocations in a high-hysteresis sample, (6) data on EBSD and retained austenite phase fraction, and (7) a detailed description of three-dimensional shape recovery of single-crystal particles.

[23] B. Fultz and J. M. Howe, Transmission Electron Microscopy and Diffractometry of Materials, Graduate Texts in Physics (Springer, Berlin, 2002).

[24] J. Jeong, M. Alfreider, R. Konetschnik, D. Kiener, and S. H. Oh, In-situ TEM observation of $\{10 \overline{1} 2\}$ twin-dominated deformation of Mg pillars, Acta Mater. 158, 407 (2018).

[25] A. Mussi, P. Cordier, S. Demouchy, and C. Vanmansart, Characterization of the glide planes of the [001] screw dislocations in olivine using electron tomography, Phys. Chem. Miner. 41, 537 (2014).
[26] S. Miyazaki, K. Otsuka, and Y. Suzuki, Transformation pseudoelasticity and deformation behavior in a Ti-50.6 at\% Ni alloy, Scr. Metall. 15, 287 (1981).

[27] G. Tan and Y. Liu, Comparative study of deformation-induced martensite stabilisation via martensite reorientation and stressinduced martensitic transformation in $\mathrm{NiTi}$, Intermetallics $\mathbf{1 2}$, 373 (2004).

[28] G. Kang, Q. Kan, L. Qian, and Y. Liu, Ratchetting deformation of super-elastic and shape-memory NiTi alloys, Mech. Mater. 41, 139 (2009).

[29] D. Dimiduk, C. Woodward, R. LeSar, and M. Uchic, Scalefree intermittent flow in crystal plasticity, Science 312, 1188 (2006).

[30] F. F. Csikor, C. Motz, D. Weygand, M. Zaiser, and S. Zapperi, Dislocation avalanches, strain bursts, and the problem of plastic forming at the micrometer scale, Science 318, 251 (2007).

[31] J. R. Greer, W. C. Oliver, and W. D. Nix, Size dependence of mechanical properties of gold at the micron scale in the absence of strain gradients, Acta Mater. 53, 1821 (2005).

[32] S. Brinckmann, J.-Y. Kim, and J. R. Greer, Fundamental Differences in Mechanical Behavior between Two Types of Crystals at the Nanoscale, Phys. Rev. Lett. 100, 155502 (2008). 